Érick Falardeau, Université Laval

Bernard Wentzel, IRDP - Neufchâtel

\section{Enjeux de savoir(s) et profession enseignante}

doi:10.18162/fp. 2016.431

Le métier d'enseignant a connu au cours des dernières décennies un processus de professionnalisation, portant sur «le passage des occupations aux professions, le passage des connaissances empiriques aux connaissances scientifiques »(Wittorski,2005). Certaines analyses ont néanmoins recensé des critiques récurrentes dans le discours des acteurs de l'enseignement, à l'égard de savoirs considérés comme trop théoriques et inappropriés, surtout pour préparer efficacement les jeunes enseignants à commencer leur carrière. Les questions énoncées par Bru (2002) n'ont rien perdu de leur pertinence dix ans après : comment réunir les conditions d'un échange plus soutenu entre savoirs de la recherche et savoirs des professionnels de l'éducation? quels types de rapports et quelles modalités de relations entre ces différents savoirs peut-on concevoir? Les contributions incluses dans ce numéro, issues d'un symposium tenu dans le cadre du colloque international du CRIFPE en mai 2013, ont pour objectif d'aborder la construction, la restitution et l'articulation entre les différents savoirs de l'enseignant professionnel, notamment à partir des thématiques suivantes: tentatives de typologisation des savoirs; tensions entre théorie et pratique en formation; intégration de la recherche et émergence du modèle réflexif; articulation et complémentarité entre savoirs à enseigner et savoirs pour enseigner. Les contributions prennent la forme de comptes rendus de recherches ou de dispositifs de formation appuyés par une théorisation des savoirs enseignés.

Dans leur article, D. Jeffrey, D. Harvengt et C. Kouassi abordent la question des savoirs professionnels sous l'angle de l'éthique. Comment la construction d'une éthique professionnelle peut-elle s'appuyer sur des savoirs théoriques plutôt que sur une éthique de la vertu, héritée des anciens modèles des écoles normales et essentiellement religieuse? F. Joliat et F. Arcidiacono s'intéressent pour leur part à l'écriture des mémoires professionnels des futurs enseignants à la HEP-BEJUNE, en Suisse.Quelle place la recherche peut-elle prendre dans la formation 
des enseignants? Comment le savoir théorique peut-il médiatiser la réflexion des stagiaires sur leur agir pédagogique? Et quels champs disciplinaires dominent dans les axes de recherche choisis par les futurs enseignants? Autant de questions qui permettent d'examiner le rôle de l'écriture réflexive dans la formation à l'enseignement. A. Stumpf, F. Joliat et D. Perrin examinent de leur côté la rhétorique des documents officiels concernant la conception de la professionnalisation. Leur étude des documents ministériels les a amenés à construire un outil méthodologique pour mener une analyse comparative de la façon dont les instituts de formation se sont approprié les modèles de professionnalisation.

Dans une perspective plus disciplinaire, M. Giglio aborde la question de la créativité dans la constitution des savoirs professionnels des enseignants. Il a étudié avec des étudiants stagiaires certains gestes de l'enseignant qui seraient nécessaires pour guider les élèves durant un acte d'apprentissage créatif et collaboratif en classe. Enfin, M. Sauvaire, M.-A. Lord et É. Falardeau présentent un dispositif de formation des enseignants de français du secondaire au Québec dans lequel les étudiants se familiarisent avec les genres de textes qu'ils auront à enseigner. L'article présente les assises théoriques de la démarche construite, soit l'écriture conçue comme une activité sociale de communication, un processus itératif qui fait appel autant à des phases de planification, de mise en texte que de révisionréécriture. La notion de genre textuel constitue le principe organisateur de cette formation, car c'est elle qui structure autant les aspects communicationnels que discursifs et langagiers des textes à produire.

\section{Références}

Wittorski, R. (Ed.) (2005). Formation, travail et professionnalisation. Paris : L'Harmattan. Collection Action et Savoir.

Bru M. (2002). Chapitre 7. Savoirs de la recherche et savoirs des praticiens de l'enseignement : jeu de dupes ou rencontre ouverte et constructive? Dans M. Bru et J. Donnay (sous la dir.), Recherches, pratiques et savoirs en éducation (pp. 133-154). Bruxelles : De Boeck Supérieur, coll. « Perspectives en éducation et formation ». doi : 10.3917/dbu. bru.2002.01.0133

\section{Pour citer cet article}

Falardeau, É. et Wentzel, B. (2016). Présentation du numéro spécial de la revue Formation et Profession « Enjeux de savoirs et profession enseignante ». Formation et profession, 24(2), 3-4. http://dx.doi.org/10.18162/fp.2016.431 